\title{
Effects of Linear Deformation on Relaxation Time and Some Fermi Properties of Metals
}

\author{
$\begin{array}{llll}\text { G.E. Adesakin } & 1 * & \begin{array}{c}\text { O.M.Osiele } \\ \text { 1.Department of Physics,Ekiti State }\end{array} & \begin{array}{l}\text { E.Bniversity, Ado-Ekiti, Nigeria } \\ \text { Uneya }\end{array}\end{array}$ \\ O. G. Edenma ${ }^{5}$ \\ 2. Department of Physics,Delta State University, Abraka, Nigeria \\ 3.Department of Computer Science,Adekunle Ajasin University, Akungba Akoko \\ 4.Department of Physics,Federal University Ote- Ekiti, Nigeria \\ 5. Department of Science Laboratory Technology, Federal Polytechnic, Auchi, Edo State, Nigeria
}

\begin{abstract}
In this work, a model for computing the relaxation time, Fermi velocity and Fermi temperature of deformed metals was developed based on free electron theory. This study generalized the work of Kiejna and Pogosov (2000) due to the shortcomings of the electron density parameter of deformed metals. They failed to account for metal dilation by assuming a constant value for the Poisson ratio of metals which leads to neglect of the uniaxial strain (deformation) in their computation. This causes the electron density parameter of both deformed and undeformed metals to be equal. The result obtained in this work revealed that there is an agreement between the experimental and computed values of the Fermi velocity, Fermi temperature and relaxation time of some of the metals calculated which shows the validity of the model used in the study. The experimental value used in this work is theoretically obtained by substituting the experimental value of Fermi energy obtained from solid state Physics by Charles Kittel (1976) into the model used in the computation. The Fermi velocity, Fermi temperature and relaxation time of all the metals subjected to different deformation decreases as deformation increases. This seems to suggest that as deformation increases the collision frequency between the interacting electron decreases which forces the relaxation time, Fermi velocity and Fermi temperature to decrease as deformation increases. This behavior could also be due to an increase in the inter-atomic spacing between the interacting electrons in the metals during deformation which reduces the strength of interaction between the electrons in metal and their-by forces the relaxation time, Fermi velocity and Fermi temperature to decrease as deformation increases.
\end{abstract}

Keywords: relaxation time, Fermi velocity, Fermi temperature, mean free path, deformation, Fermi surface

DOI: $10.7176 /$ JNSR/9-8-04

Publication date: April $30^{\text {th }} 2019$

\subsection{Introduction}

Metals achieve structural stability by letting their valence electrons roam freely through the crystal lattice. These valence electrons are the equivalents of the molecules of an ordinary gas. It is assumed that the electrons are moving about at random and colliding frequently with the residual ions (Pillai, 2010). Deformation can be described as change in shape or size of an object due to an applied stress (force) or strain. Metals could be deformed by a compressive, elongative and torsion/twisting force (Backofen, 1972). Fermi surface is the surface of constant energy $E_{F}$ in $\mathrm{k}$-space. The Fermi surface separates the unfilled from the filled orbital at absolute zero (Busch and Schade, 1976). Fermi level describe the top of the collection of electron energy levels at absolute zero temperature (Madelung, 1995) . Fermi temperature is the temperature at which thermal effects are comparable to quantum effects associated with Fermi statistics (Ashcroft and Mermin,1976). Relaxation time is determined by the rate at which the shifted distribution returns to equilibrium (Pines, 1964). Relaxation time represents the effect of collisions between electrons and ions or impurities on electrical conduction in a metal (Madelung, 1995). The conductivity of a material is directly proportional to relaxation time and highly conductive materials have relatively long relaxation time (Busch and Schade, 1976). Mean free path is the average distance traveled by a moving particle (such as an atom, a molecule, a photon) between successive impacts (collisions), which modify its direction, energy and other particle properties (Ashcroft and Mermin, 1976).

The electrical properties of metal are determined by the shape of the Fermi Surface as the current is due to changes in the occupancy of states near the Fermi Surface (Kittel, 1976). The concept of Fermi surface enables us to visualize the relative fullness or occupation of the allowed empty lattice bands geometrically in $\mathrm{k}$-space and thus helps in the theoretical determination of the electronic properties of metal, semiconductor or an insulator (Pines, 1964). The purpose of the Fermi surface construction is to know about the details of the motion of an itinerant electron in solid (Kachhava, 1992). The Fermi energy is determined by means of the neutrality condition that has a precisely defined position between the valence and conduction band (Elliott, 1997).

For the properties of metals the topology of the Fermi surface is very important, the electrical conductivity, thermal conductivity and many other properties are essentially determined by only the relatively few electrons whose energies are in the range where the external force is small, because of the Pauli principle only those electrons can change their states (Animalu, 1977). 
Consequently, a lot of efforts have been made to study the effects of deformation on some properties of selected metals. Adeshakin and Osiele (2012) developed a model for computing the surface energy and surface stress of deformed metals based on the structureless pseudo potential formalism. The developed models were tested by using them to compute the surface energy and surface stress of different classes of metals for different values of strain deformation. The results obtained revealed that deformation causes a reduction of surface energy and this reduction in surface energy is more pronounced in simple and alkaline metals. For surface stress of deformed metals, tensile stress is present in most metallic surfaces, although a few metals possess compressive stress on their surfaces. In the presence of deformation, the surface stress of some metals decreases. For Ti and Pt, deformation causes an increase in their surface stress, while deformation causes an increase in the surface stress of Mo and $\mathrm{W}$ causing the stress on their surfaces to change from compressive to tensile. But for $\mathrm{Cr}, \mathrm{Be}$ and $\mathrm{Al}$, the stress on their surfaces changes depending on the amount of deformation. Adeshakin et al. (2012), developed a model based on the structureless pseudopotential to compute the correlation, binding and cohesive energy of deformed and undeformed metals. The computed binding and cohesive energy of metals were compared with available experimental values. The results obtained showed that correlation energy increases with increase in electron density parameter. The computed binding energy and cohesive energy of metals were in good agreement with experimental values. The results obtained also showed that deformation causes a decrease in the binding energy of metals and it does not cause a significant change in the cohesive energy of metals, although transition metals have high values of cohesive energy compared to alkaline and simple metals. Keijna and Pogosov, (2000) experimentally investigated the effect of deformation on some electronic properties of metals by measuring directly a sample of deformed metals using Kelvin method. They observed that the contact potential difference of metals increase/decreases when compressed/tensed. Shore and Rose (1991), calculated the surface properties of metals. They used an ideal-metal model. The results of the calculated surface properties are in agreement with experimental results but have slightly less satisfactory agreement for bulk properties than that of the structureless pseudopotential model of Perdew et al (1990). Kiejna and Pogosov (1999), theoretically determined the effect of deformation on the surface properties of metal, based on stabilized jellium model, and derived modified equations for the stabilization energy of the deformed Wigner Seitz cell as a function of the bulk electron density and the given deformation. They used the electron density parameter, Poisson ratio and young's modulus of the metals as the input parameter. The results obtained were in agreement with experimental results. Pogosov and Shtepa (2006), calculated the surface stress and the contact potential difference of elastically deformed metals based on structureless pseudopotential model using self-consistent Kohn Sham method. The results of surface stress obtained were in agreement with experimental results, and also confirmed that the contact potential difference obtained for the deformed metallic surfaces by Kelvin method correspond to change in surface potential. Osiele and Edenma (2009), developed a model to compute the bulk modulus and kinetic energy contribution to the bulk modulus of metals based on structureless pseudopotential model. The computed bulk modulus of metals were in good agreement with experimental values for metal in low density limit and the agreement between the computed and experimental bulk modulus of metals decreases towards the high density limit. The results also revealed that the kinetic energy contributes significantly to the bulk modulus of metals. Osiele and Olubosede (2007) developed a stabilized jellium model to calculate the surface stress and face dependent surface stress of metals. The surface stress of metals, calculated using the stabilized jellium model, was compared with results obtained using other methods and available experimental values. The results obtained revealed that tensile stress is present on the surfaces of the metals, and metals in the high density limit have high strain derivative and high values of surface stress while metals in the low density limit have low strain derivatives and low values of surface stress.

This study generalized the work of Kiejna and Pogosov (2000) due to the shortcomings of the electron density parameter of deformed metals. They failed to account for metal dilation by assuming a constant value for the Poisson ratio of metals which leads to neglect of the uniaxial strain in their computation. In this work the poisson ratio relating the compression to elongation in the direction of applied deformation is taking into consideration while computing the electron density parameter for deformed metals. The Relaxation time, Fermi velocity and Fermi Temperature of metals consisting of monovalent, divalent, trivalent, noble and transition metals were computed for deformed metals. This will enable us to predict the properties of deformed metals and how this property of metals varies with deformation. The metals used in this study were chosen based on the availability of experimental data, their industrial and technological applications, and availability of some physical constants of metals that is required for computation.

\subsection{Theoretical Consideration}

Considering a hypothetical crystal in the shape of a rectangular parallelepiped. In the undeformed state all of its faces are equivalent. Assume that deformation is a measured quantity and a metallic crystal to be considered as assembled from a number of simple crystallites. Express the average electron density in a metal as a function of deformation. Express the average electron density in a metal as a function of deformation for this purpose, consider 
a cubic cell of the side length $a_{o}$ and volume (Kiejna and Pogosov, 1999)

$\Omega_{0}=a_{0}^{3}=\frac{4}{3} \pi r_{0}^{3}$

where $r_{o}$ is the radius of the Wigner-Seitz cell given as $r_{0}=z^{\frac{1}{3}} r_{s}$ where $r_{s}$ is the electron density parameter of undeformed metal defined as the radius of sphere containing one electron on average and a measure of the average distance between electrons. $r_{s}$ is defined as

$r_{s}=\left(\frac{3}{4} \pi n\right)^{1 / 3}$

where $\mathrm{n}$ is the electronic density of undeformed crystal. For a cubic cell deformed by applying an elongative force along the $\mathrm{x}$-axis, the volume of the deformed cell is

$\Omega_{d}=a_{x} a_{y}^{2}=\frac{4}{3} \pi a b^{2}$

where $a_{x}, a_{y}=a_{z}$ are the sides of the deformed cubic cell. If the uniaxial strain is $u_{x x}$, then $a_{x}=a_{0}\left[1+u_{x x}\right]$

$a_{z}=a_{0}\left[1+u_{z z}\right]=a_{0}\left[1-v u_{x x}\right]$

where $v$ is the polycrystalline Poisson ratio that relates the transversal compression to the elongation in the direction of the applied deformation that is $u_{y y}=u_{z z}=-v u_{x x}$

The ratio of the unit volume of the deformed cubic cell to that of the undeformed cell is $\frac{\Omega_{d}}{\Omega_{0}}=\frac{a_{0}\left[1+u_{x x}\right] a_{0}\left[1+u_{y y}\right] a_{0}\left[1+u_{z z}\right]}{a_{0}^{3}}$

$\frac{\Omega_{d}}{\Omega_{0}}=\left[1+u_{x x}\right]\left[1+u_{y y}+u_{z z}+u_{y y} u_{z z}\right]$

Neglecting higher order terms of the uniaxial strain, then (Kiejna and Pogosov,

$$
\frac{\Omega_{d}}{\Omega_{0}}-1=u_{x x}+u_{y y}+u_{z z}
$$

From equation (4), then for the deformed cube,

$a=r_{0}\left(1+u_{x x}\right)$

$b=r_{0}\left(1-v u_{x x}\right)$

The average electron density in the deformed metal is

$n_{a v}=\frac{n_{0} \Omega_{0}}{\Omega}=\frac{n_{0} a_{0}^{3}}{a_{0}\left(1+u_{x x}\right)\left(a_{0}\left(1-v u_{x x}\right)\right)^{2}}$

$n_{a v}=n_{0}\left[1-(1-2 v) u_{x x}\right]+0\left(u_{x x}^{2}\right)$

The electron gas parameter of the deformed metal is obtained from its volume as

$\frac{4}{3} \pi r_{s u}^{3}=\frac{4}{3} \pi a b^{2}$

$r_{s u}^{3}=r_{0}\left(1+u_{x x}\right)\left(r_{0}^{2}\left(1-v u_{x x}\right)^{2}\right)$

$r_{s u}^{3}=r_{0}^{3}\left(1+u_{x x}-v u_{x x}+v u_{x x}^{2}-v u_{x x}-v u_{x x}^{2}\right)$

Neglecting higher order terms in the strain or deformation, we have (Kiejna and Pogosov, 1999)

$r_{s u}=r_{0}\left(1+u_{x x}(1-2 v)\right)^{1 / 3}$

The electron gas parameter of deformed metals, $\mathrm{r}_{\mathrm{su}}$ gives the mean inter electronic distance in a deformed metal (Kiejna and Pogosov, 1999)

To derive the concept of the Fermi surface, we pour electron into our empty lattice. These will fill one constant energy surface after another until all the electrons are used up. The Fermi surface is the boundary between 
filled and empty states in k-space in the ground state of the crystal. If the potential seen by each valence electron as it moves through the crystal is constant, the electrons then fall automatically into the plane wave states (Kachhava, 1992).

$|k|=\psi_{k}-e^{i k \cdot r}$

with energy

$$
E=\frac{\hbar^{2} k^{2}}{2 m}=\frac{\hbar^{2}}{2 m}\left(k_{x}^{2}+k_{y}^{2}+k_{z}^{2}\right)
$$

To satisfy the Pauli principle, we fill the states up to a wave number $\mathrm{k}_{\mathrm{F}}$ called the Fermi radius given (Kachhava, 1992) by

$\frac{2}{(2 \pi)^{3}} \int_{o}^{k_{F}} d k=n z$

where $\mathrm{n}$ is the electronic concentration and $\mathrm{z}$ is the number of valence electron.

The Fermi energy $E_{F}$ is the energy of the highest filled state in the highest filled energy band which contains electrons in a metal at absolute zero. The Fermi energy $E_{F}$ is defined as

$E_{F}=\frac{\hbar^{2}{k_{F}}^{2}}{2 m}$

where $\hbar$ is the normalized planks constrant, and $m$ is the electron mass.

Using the concept of Fermi-Dirac distribution (Kittel, 1976)

$f=\frac{1}{\exp \left[\left(E-E_{F}\right) / K_{B} T\right]+1}$

in which case $E_{F}$ is the energy for which the Fermi-Dirac distribution function is half.

For $K_{B} T \ll E_{F}$, the temperature variation of the Fermi energy is given by

$E_{F}(T)$

$=E_{F}\left[1-\frac{\pi^{2}}{12}\left(\frac{k_{B} T}{E_{F}}\right)^{2}\right]$

Inserting equation (14) into equation (15), then

$$
\begin{aligned}
E_{F}=\frac{\hbar^{2}}{2 m}\left(\frac{3 \pi^{2} z}{\Omega_{0}}\right)^{\frac{2}{3}} & \\
& =\frac{\hbar^{2}}{2 m}\left(3 \pi^{2} n z\right)^{\frac{2}{3}}
\end{aligned}
$$

where $\Omega_{0}$ is the Fermi characteristics in terms of the electron density parameter $r_{s}$ defined by

$\Omega_{0}=\left(\frac{4 \pi}{3}\right) r_{s}^{3} z$.

The Fermi radius is given as(Kachhava, 1992)

$k_{F}=\left(\frac{9 \pi}{4}\right)^{1 / 3} \frac{1}{r_{s}}$

The differential transport equation can be written for electrons in the form:

$$
\frac{d f}{d t}=\frac{\partial f}{\partial t}+\vec{V} \cdot \nabla f+\frac{1}{\hbar} \vec{F} \cdot \nabla_{\vec{k}} f=\left(\frac{\partial f}{\partial t}\right)_{\text {scatt }}
$$

where $\vec{V}=d \vec{r} / d t$ is the rate of change of position on the trajectory for a given momentum, and $\vec{F}=\hbar \frac{d \vec{k}}{d t}$ is the rate of change of momentum with time at a given point.

If the electric field is applied at time $\mathrm{t}=0$ to a filled Fermi sphere centered at the origin of $\mathrm{k}$-space, then the relaxation time $\tau$ is

$$
\tau=\frac{\Lambda}{V_{F}}
$$

where $\Lambda$ is the deformed mean free path of a conduction electron and is obtained as

$$
\Lambda=\frac{2 \pi^{2} \hbar^{2}}{m e}\left(\frac{3}{16 \pi z}\right)^{2 / 3} \frac{1}{\left[r_{o}\left(1+u_{x x}(1-2 v)\right)^{1 / 3}\right]^{2}}
$$

and $V_{F}$ is the Fermi Velocity of the conduction electron obtained as

$$
V_{F}=\frac{\hbar}{m e}\left(\frac{9 \pi}{4}\right)^{\frac{1}{3}} \frac{1}{r_{o}\left(1+u_{x x}(1-2 v)\right)^{1 / 3}}
$$

The obtained expression for the Fermi temperature is

$$
T_{F}=\frac{\hbar}{2 m e k_{B}}\left(3 \pi^{2} n\right)^{\frac{2}{3}}
$$


where is the average electron density given by equation (8)

In this work, Relaxation time, Fermi velocity and Fermi Temperature of deformed metals were computed using equation (29), (31) and (33), and how deformation affects these properties of metals are studied.

\subsection{Results and Discussion}

\subsection{Effect of Deformation on Fermi Velocity}

Figure 1 shows the variation of Fermi velocity with electron gas parameter for some elemental metals consisting of monovalent, divalent, trivalent and polyvalent metals. The trend exhibited by Fermi velocity of metals in Figure 1 revealed that the Fermi velocity of metals decreases with an increase in electron density parameter with the metals in the high density region having high Fermi velocity while metals in the low density region were having low Fermi velocity. This may be due to the fact that metals in the high density region were having high valence electron density while metals in the low density region were having low valence electron density. The trend exhibited by metals in figure 1 also revealed that the Fermi velocity of metals depend on the electronic concentration as metals in the high density region were having high Fermi velocity. Figure 1 also revealed that there is an agreement between the experimental and computed values of the Fermi velocity of some of the metals calculated which shows the validity of the model used in the study. The experimental value used in this work is theoretically obtained by substituting the experimental value of Fermi energy obtained from solid state Physics by Charles Kittel (1976) into the model used in the computation of the Fermi velocity in this work. Figure 2 shows the variation of Fermi velocity with deformation for some elemental metals of different groups and periods. Figure 2 revealed that the Fermi velocity of metals decreases as deformation increases with metals in the high density region having high Fermi velocity while metals in the low density region has low Fermi velocity when subjected to different deformation. Table 1 revealed that molybdenum and tungsten has the highest Fermi velocity while potassium has the lowest Fermi velocity among all the metals subjected to different deformation. This seems to suggest that the lower the electronic concentration in metal the higher the effect of deformation on the Fermi velocity of the metal and the higher the electronic concentration and density of valence electron in metal the lower the effect of deformation on the metal. The trend exhibited by metals in figure 2 could also be due to the fact that as deformation increases the temperature and mean free path between the interacting electrons in metals also increases which causes the frequency of collision between the interacting electrons to decrease and their by forces the Fermi velocity to decrease as deformation increases. The trend exhibited by metals in Figure 2 revealed that the Fermi velocity of metal is greatly affected by deformation.

\subsection{Effect of Deformation on Relaxation Time}

Figure 3 shows the variation of relaxation time with electron gas parameters for some elemental metals containing monovalent, divalent, trivalent and polyvalent metals. Figure 3 revealed that the relaxation time of most of the metals is concentrated in the high density region this could be due to the fact that relaxation time depend on electronic concentration. Figure 3 also revealed that there is an agreement between the experimental and computed values of the relaxation time this indicate that the model used in the study is useful in the theoretical prediction of relaxation time of metals. The experimental value of the relaxation time is theoretically obtained by substituting the experimental values obtained for Fermi temperature and Fermi velocity into the model obtained for the computation of the relaxation time of metals. The trend exhibited by metals in figure 3 also revealed that metals with high conductive properties have relatively long relaxation time.

Figure 4 shows the variation of relaxation time with deformation for some elemental metals of different groups and periods. Figure 4 revealed that the relaxation time of all the metals investigated decreases as the deformation increases with the metals in the high density region having high relaxation time while metals in the low density region have low relaxation time. This seems to suggest that as deformation increases the collision between the interacting electron decreases due to increase in the interatomic spacing and vibrations between the different interacting electrons which forces the relaxation time to decrease as deformation increases in the metals. Also, the decrease in the relaxation time as deformation increases could also be due to lattice imperfection, intrinsic excitation, reduction in thermal and electrical properties which affect the density of conducting electron and theirby forces the relaxation time to decrease as deformation increases. Table 2 revealed that Tantalum has the lowest relaxation time while Bismuth has the highest relaxation time among all the metals subjected to different deformation. This trend revealed that the relaxation time defined as the reciprocal of collision probability per unit time depend on the density of the valence electron in a metal. The trend exhibited by metals in Figure 4 revealed that the relaxation time of metal is greatly affected by deformation.

\subsection{Effect of Deformation on Fermi Temperature}

Figure 5 shows the variation of Fermi temperature of metals with electron gas parameter for some elemental metals containing monovalent, divalent, trivalent and polyvalent metals. Figure 5 reveals that the Fermi temperature decreases with an increase in the electron gas parameter, we also observed that the Fermi temperature of most of 
the metals is more concentrated in the high density region. There is an agreement between the computed and experimental value of the Fermi temperature which revealed that the model take into consideration most of the properties that the Fermi temperature of metals depend upon. The experimental value of the Fermi temperature used in this work is theoretically obtained by substituting the experimental value of the Fermi energy of metals in solid state Physics by Charles Kittel, (1976) into the model obtained for the computation of the Fermi temperature of metals. The trend exhibited by metals in figure 5 seems to suggest that the higher the density of mobile charge in metals the higher the Fermi temperature of the metals.

Figure 6 shows the variation of Fermi temperature with strain for different elemental metals belonging to different groups and period. Figure 6 revealed that the Fermi temperature of metals decreases with an increase in deformation with metals in the high density region having high Fermi temperature, while metal in the low density region has low Fermi temperature. This could be due to the fact that as deformation increases there is change in phase and electronic band structure of the electrons in metals which causes some disorderliness and their-by reduces the strength of interaction between the electron and then forces the Fermi temperature to decrease as deformation increases. The trend exhibited by metals in Figure 6 revealed that the Fermi temperature of metals is greatly affected by deformation. Table 3 revealed that potassium has the lowest Fermi temperature while chromium has the highest Fermi temperature among all the metals subjected to different deformation. This seems to suggest that the higher the electronic concentration of metals the lower the effect of deformation on the Fermi temperature of the metals and the lower the electronic concentration of metals the higher the effect of deformation on the Fermi temperature of the metal.

Table 1: Fermi Velocity of Deformed Metals (Hartree)

\begin{tabular}{|l|l|l|l|l|l|l|l|l|l|l|}
\hline & & \multicolumn{9}{|c|}{ Strain } \\
\hline METALS & r $_{\text {(a.u) }}$ & $\mathbf{0 . 2}$ & $\mathbf{0 . 4}$ & $\mathbf{0 . 6}$ & $\mathbf{0 . 8}$ & $\mathbf{1 . 0}$ & $\mathbf{1 . 2}$ & $\mathbf{1 . 4}$ & $\mathbf{1 . 6}$ & $\mathbf{1 . 8}$ \\
\hline $\mathrm{K}$ & 4.96 & 0.376 & 0.364 & 0.353 & 0.344 & 0.335 & 0.327 & 0.320 & 0.314 & 0.308 \\
\hline $\mathrm{Cu}$ & 2.67 & 0.695 & 0.672 & 0.652 & 0.634 & 0.619 & 0.604 & 0.591 & 0.579 & 0.568 \\
\hline $\mathrm{Ag}$ & 3.02 & 0.615 & 0.595 & 0.577 & 0.562 & 0.548 & 0.535 & 0.523 & 0.512 & 0.503 \\
\hline $\mathrm{Be}$ & 1.87 & 0.989 & 0.956 & 0.928 & 0.903 & 0.880 & 0.859 & 0.841 & 0.824 & 0.808 \\
\hline $\mathrm{Mg}$ & 2.65 & 0.700 & 0.677 & 0.657 & 0.639 & 0.623 & 0.609 & 0.595 & 0.583 & 0.572 \\
\hline $\mathrm{Cr}$ & 1.86 & 0.994 & 0.962 & 0.933 & 0.908 & 0.885 & 0.864 & 0.845 & 0.828 & 0.812 \\
\hline $\mathrm{Fe}$ & 2.12 & 0.873 & 0.845 & 0.820 & 0.797 & 0.777 & 0.759 & 0.743 & 0.727 & 0.713 \\
\hline $\mathrm{Ni}$ & 2.07 & 0.894 & 0.865 & 0.839 & 0.816 & 0.796 & 0.777 & 0.760 & 0.745 & 0.730 \\
\hline $\mathrm{Zn}$ & 2.31 & 0.802 & 0.776 & 0.753 & 0.732 & 0.714 & 0.697 & 0.682 & 0.668 & 0.655 \\
\hline $\mathrm{Cd}$ & 2.59 & 0.716 & 0.693 & 0.672 & 0.654 & 0.637 & 0.623 & 0.609 & 0.597 & 0.585 \\
\hline $\mathrm{Al}$ & 2.07 & 0.894 & 0.864 & 0.839 & 0.816 & 0.796 & 0.777 & 0.760 & 0.745 & 0.730 \\
\hline $\mathrm{Bi}$ & 2.25 & 0.823 & 0.796 & 0.773 & 0.752 & 0.733 & 0.716 & 0.700 & 0.686 & 0.673 \\
\hline $\mathrm{Ti}$ & 1.92 & 0.963 & 0.932 & 0.904 & 0.879 & 0.857 & 0.837 & 0.819 & 0.802 & 0.787 \\
\hline $\mathrm{Y}$ & 2.61 & 0.711 & 0.688 & 0.667 & 0.649 & 0.633 & 0.618 & 0.604 & 0.592 & 0.581 \\
\hline $\mathrm{Sn}$ & 2.22 & 0.834 & 0.807 & 0.783 & 0.762 & 0.743 & 0.725 & 0.709 & 0.695 & 0.682 \\
\hline $\mathrm{Pb}$ & 2.30 & 0.806 & 0.779 & 0.756 & 0.735 & 0.717 & 0.700 & 0.685 & 0.671 & 0.658 \\
\hline $\mathrm{Mo}$ & 1.61 & 1.147 & 1.109 & 1.076 & 1.047 & 1.021 & 0.997 & 0.975 & 0.955 & 0.937 \\
\hline $\mathrm{W}$ & 1.62 & 1.140 & 1.102 & 1.070 & 1.041 & 1.014 & 0.991 & 0.969 & 0.949 & 0.931 \\
\hline $\mathrm{Au}$ & 2.39 & 0.776 & 0.750 & 0.728 & 0.708 & 0.690 & 0.674 & 0.659 & 0.645958 & 0.634 \\
\hline $\mathrm{Pt}$ & 2.00 & 0.925 & 0.895 & 0.868 & 0.845 & 0.823 & 0.804 & 0.787 & 0.771 & 0.756 \\
\hline $\mathrm{Ta}$ & 2.84 & 0.654 & 0.632 & 0.614 & 0.597 & 0.582 & 0.568 & 0.556 & 0.545 & 0.534 \\
\hline
\end{tabular}


Table 2: Relaxation Time of Deformed Metals (Hartree)

\begin{tabular}{|l|l|l|l|l|l|l|l|l|l|l|}
\hline & \multicolumn{9}{|c|}{ Strain } \\
\hline Metals & $\begin{array}{l}\text { rs } \\
(\text { a.u) }\end{array}$ & $\mathbf{0 . 2}$ & $\mathbf{0 . 4}$ & $\mathbf{0 . 6}$ & $\mathbf{0 . 8}$ & $\mathbf{1 . 0}$ & $\mathbf{1 . 2}$ & $\mathbf{1 . 4}$ & $\mathbf{1 . 6}$ & $\mathbf{1 . 8}$ \\
\hline $\mathrm{K}$ & 4.96 & 0.308 & 0.298 & 0.289 & 0.281 & 0.273 & 0.267 & 0.261 & 0.255 & 0.250 \\
\hline $\mathrm{Cu}$ & 2.67 & 0.575 & 0.556 & 0.539 & 0.524 & 0.511 & 0.499 & 0.488 & 0.478 & 0.468 \\
\hline $\mathrm{Ag}$ & 3.02 & 0.508 & 0.491 & 0.476 & 0.463 & 0.541 & 0.440 & 0.431 & 0.422 & 0.413 \\
\hline $\mathrm{Be}$ & 1.87 & 0.522 & 0.504 & 0.489 & 0.476 & 0.463 & 0.452 & 0.442 & 0.433 & 0.425 \\
\hline $\mathrm{Mg}$ & 2.65 & 0.367 & 0.355 & 0.344 & 0.334 & 0.326 & 0.318 & 0.311 & 0.305 & 0.299 \\
\hline $\mathrm{Cr}$ & 1.86 & 0.525 & 0.507 & 0.492 & 0.478 & 0.466 & 0.455 & 0.445 & 0.435 & 0.427 \\
\hline $\mathrm{Fe}$ & 2.12 & 0.460 & 0.444 & 0.431 & 0.419 & 0.408 & 0.398 & 0.390 & 0.381 & 0.374 \\
\hline $\mathrm{Ni}$ & 2.07 & 0.471 & 0.455 & 0.441 & 0.429 & 0.418 & 0.408 & 0.399 & 0.391 & 0.383 \\
\hline $\mathrm{Zn}$ & 2.31 & 0.422 & 0.407 & 0.395 & 0.384 & 0.374 & 0.365 & 0.357 & 0.350 & 0.343 \\
\hline $\mathrm{Cd}$ & 2.59 & 0.376 & 0.363 & 0.352 & 0.342 & 0.333 & 0.326 & 0.318 & 0.312 & 0.306 \\
\hline $\mathrm{Al}$ & 2.07 & 0.360 & 0.348 & 0.338 & 0.328 & 0.320 & 0.312 & 0.305 & 0.299 & 0.293 \\
\hline $\mathrm{Bi}$ & 2.25 & 0.684 & 0.661 & 0.641 & 0.623 & 0.607 & 0.593 & 0.580 & 0.568 & 0.556 \\
\hline $\mathrm{Ti}$ & 1.92 & 0.389 & 0.376 & 0.364 & 0.354 & 0.345 & 0.337 & 0.330 & 0.323 & 0.316 \\
\hline $\mathrm{Y}$ & 2.61 & 0.285 & 0.276 & 0.267 & 0.260 & 0.253 & 0.247 & 0.242 & 0.237 & 0.232 \\
\hline $\mathrm{Sn}$ & 2.22 & 0.278 & 0.268 & 0.260 & 0.253 & 0.247 & 0.241 & 0.235 & 0.230 & 0.226 \\
\hline $\mathrm{Pb}$ & 2.30 & 0.268 & 0.259 & 0.251 & 0.244 & 0.238 & 0.232 & 0.227 & 0.222 & 0.218 \\
\hline $\mathrm{Mo}$ & 1.61 & 0.294 & 0.284 & 0.276 & 0.268 & 0.261 & 0.255 & 0.249 & 0.244 & 0.239 \\
\hline $\mathrm{W}$ & 1.62 & 0.329 & 0.318 & 0.309 & 0.300 & 0.293 & 0.286 & 0.279 & 0.273 & 0.268 \\
\hline $\mathrm{Au}$ & 2.39 & 0.222 & 0.215 & 0.208 & 0.203 & 0.198 & 0.193 & 0.189 & 0.185 & 0.181 \\
\hline $\mathrm{Pt}$ & 2.00 & 0.309 & 0.298 & 0.289 & 0.281 & 0.274 & 0.267 & 0.262 & 0.256 & 0.251 \\
\hline $\mathrm{Ta}$ & 2.84 & 0.150 & 0.145 & 0.140 & 0.136 & 0.133 & 0.130 & 0.127 & 0.124 & 0.122 \\
\hline
\end{tabular}

Table 3: Fermi Temperature of Deformed Metals (Hartree)

\begin{tabular}{|l|l|l|l|l|l|l|l|l|l|l|}
\hline & & \multicolumn{9}{|c|}{ Strain } \\
\hline Metals & rs (a.u) & $\mathbf{0 . 2}$ & $\mathbf{0 . 4}$ & $\mathbf{0 . 6}$ & $\mathbf{0 . 8}$ & $\mathbf{1 . 0}$ & $\mathbf{1 . 2}$ & $\mathbf{1 . 4}$ & $\mathbf{1 . 6}$ & $\mathbf{1 . 8}$ \\
\hline $\mathrm{K}$ & 4.96 & 0.0709 & 0.0663 & 0.0624 & 0.0591 & 0.0561 & 0.0535 & 0.0512 & 0.0492 & 0.0473 \\
\hline $\mathrm{Cu}$ & 2.67 & 0.242 & 0.226 & 0.213 & 0.201 & 0.191 & 0.182 & 0.175 & 0.168 & 0.161 \\
\hline $\mathrm{Ag}$ & 3.02 & 0.189 & 0.177 & 0.167 & 0.158 & 0.150 & 0.143 & 0.137 & 0.131 & 0.126 \\
\hline $\mathrm{Be}$ & 1.87 & 0.489 & 0.457 & 0.431 & 0.407 & 0.387 & 0.369 & 0.353 & 0.339 & 0.326 \\
\hline $\mathrm{Mg}$ & 2.65 & 0.245 & 0.229 & 0.216 & 0.204 & 0.194 & 0.185 & 0.177 & 0.170 & 0.164 \\
\hline $\mathrm{Cr}$ & 1.86 & 0.494 & 0.462 & 0.435 & 0.412 & 0.391 & 0.373 & 0.357 & 0.343 & 0.330 \\
\hline $\mathrm{Fe}$ & 2.12 & 0.381 & 0.357 & 0.336 & 0.318 & 0.302 & 0.288 & 0.276 & 0.265 & 0.254 \\
\hline $\mathrm{Ni}$ & 2.07 & 0.400 & 0.374 & 0.352 & 0.333 & 0.317 & 0.302 & 0.289 & 0.277 & 0.267 \\
\hline $\mathrm{Zn}$ & 2.31 & 0.322 & 0.301 & 0.283 & 0.268 & 0.255 & 0.243 & 0.233 & 0.223 & 0.215 \\
\hline $\mathrm{Cd}$ & 2.59 & 0.256 & 0.240 & 0.226 & 0.214 & 0.203 & 0.194 & 0.185 & 0.178 & 0.171 \\
\hline $\mathrm{Al}$ & 2.07 & 0.400 & 0.374 & 0.352 & 0.333 & 0.317 & 0.302 & 0.289 & 0.277 & 0.267 \\
\hline $\mathrm{Bi}$ & 2.25 & 0.339 & 0.317 & 0.299 & 0.282 & 0.268 & 0.256 & 0.245 & 0.235 & 0.226 \\
\hline $\mathrm{Ti}$ & 1.92 & 0.464 & 0.434 & 0.409 & 0.387 & 0.368 & 0.351 & 0.335 & 0.322 & 0.310 \\
\hline $\mathrm{Y}$ & 2.61 & 0.253 & 0.236 & 0.223 & 0.211 & 0.200 & 0.191 & 0.183 & 0.175 & 0.169 \\
\hline $\mathrm{Sn}$ & 2.22 & 0.348 & 0.326 & 0.307 & 0.290 & 0.276 & 0.263 & 0.252 & 0.241 & 0.232 \\
\hline $\mathrm{Pb}$ & 2.30 & 0.324 & 0.304 & 0.286 & 0.270 & 0.257 & 0.245 & 0.235 & 0.225 & 0.217 \\
\hline $\mathrm{Mo}$ & 1.61 & 0.657 & 0.615 & 0.579 & 0.548 & 0.521 & 0.497 & 0.475 & 0.456 & 0.439 \\
\hline $\mathrm{W}$ & 1.62 & 0.649 & 0.608 & 0.572 & 0.541 & 0.514 & 0.491 & 0.470 & 0.451 & 0.433 \\
\hline $\mathrm{Au}$ & 2.39 & 0.301 & 0.281 & 0.265 & 0.251 & 0.238 & 0.227 & 0.217 & 0.209 & 0.201 \\
\hline $\mathrm{Pt}$ & 2.00 & 0.428 & 0.400 & 0.377 & 0.357 & 0.339 & 0.323 & 0.309 & 0.297 & 0.286 \\
\hline $\mathrm{Ta}$ & 2.84 & 0.214 & 0.200 & 0.188 & 0.178 & 0.169 & 0.161 & 0.155 & 0.148 & 0.143 \\
\hline
\end{tabular}


Table 4: Fermi temperature, Fermi Velocity and Relaxation time of Undeformed Metals

\begin{tabular}{|l|l|l|l|l|l|l|l|}
\hline $\begin{array}{l}\text { Metal } \\
\text { S }\end{array}$ & $\begin{array}{l}\text { Electron } \\
\text { Density } \\
\text { Paramete } \\
\text { r rs(a.u) }\end{array}$ & $\begin{array}{l}\text { Experiment } \\
\text { al Fermi } \\
\text { Temperatur } \\
\text { e (Hartree) }\end{array}$ & $\begin{array}{l}\text { Computed } \\
\text { Fermi } \\
\text { Temperatur } \\
\text { e (Hartree) }\end{array}$ & $\begin{array}{l}\text { Experiment } \\
\text { al Fermi } \\
\text { Velocity } \\
\text { (Hartree) }\end{array}$ & $\begin{array}{l}\text { Compute } \\
\text { d Fermi } \\
\text { Velocity } \\
\text { (Hartree) }\end{array}$ & $\begin{array}{l}\text { Experiment } \\
\text { al } \\
\text { Relaxation } \\
\text { Time } \\
\text { (Hartree) }\end{array}$ & $\begin{array}{l}\text { Compute } \\
\text { d } \\
\text { Relaxatio } \\
\text { n Time } \\
\text { (Hartree) }\end{array}$ \\
\hline $\mathrm{K}$ & 4.96 & 5.6456 & 5.4246 & 4.1360 & 4.0542 & 2.5195 & 1.0876 \\
\hline $\mathrm{Cu}$ & 2.67 & 1.8641 & 1.8720 & 7.5155 & 7.5314 & 1.6625 & 2.0358 \\
\hline $\mathrm{Ag}$ & 3.02 & 1.4593 & 1.4632 & 6.6496 & 6.6585 & 2.5408 & 1.8002 \\
\hline $\mathrm{Be}$ & 1.87 & 3.7655 & 3.8163 & 1.0682 & 1.0753 & 2.9918 & 1.8359 \\
\hline $\mathrm{Mg}$ & 2.65 & 1.8987 & 1.9004 & 7.5849 & 7.5882 & 6.4410 & 1.2993 \\
\hline $\mathrm{Cr}$ & 1.86 & & 3.8575 & & 1.0811 & 7.4558 & 1.8448 \\
\hline $\mathrm{Fe}$ & 2.12 & 2.9560 & 2.9693 & 9.4639 & 9.4853 & 1.4437 & 1.6135 \\
\hline $\mathrm{Ni}$ & 2.07 & & 3.1145 & & 9.7144 & 1.8841 & 1.6601 \\
\hline $\mathrm{Zn}$ & 2.31 & 2.9293 & 2.5010 & 9.4212 & 8.7051 & 3.0944 & 1.4831 \\
\hline $\mathrm{Cd}$ & 2.59 & 1.9866 & 1.9894 & 7.7585 & 7.7640 & 3.5615 & 1.3162 \\
\hline $\mathrm{Al}$ & 2.07 & 3.0971 & 3.1145 & 9.6872 & 9.7144 & 4.8091 & 1.2649 \\
\hline $\mathrm{Bi}$ & 2.25 & 2.6364 & 2.6361 & 8.9377 & 8.9372 & 1.4551 & 2.4196 \\
\hline $\mathrm{Ti}$ & 1.92 & & 3.6202 & & 1.0473 & 2.4182 & 1.3563 \\
\hline $\mathrm{Y}$ & 2.61 & & 1.9591 & & 7.7045 & 4.4898 & 1.0036 \\
\hline $\mathrm{Sn}$ & 2.22 & 2.6710 & 2.7079 & 8.9962 & 9.0580 & 1.4790 & 9.7514 \\
\hline $\mathrm{Pb}$ & 2.30 & 2.4953 & 2.5228 & 8.6952 & 8.7429 & 8.6753 & 9.3982 \\
\hline $\mathrm{Mo}$ & 1.61 & & 5.1485 & & 1.2490 & 1.1716 & 1.0229 \\
\hline $\mathrm{W}$ & 1.62 & & 5.0851 & & 1.2413 & 1.1936 & 1.1494 \\
\hline $\mathrm{Au}$ & 2.39 & 1.4673 & 2.3363 & 6.6678 & 8.4140 & 9.2271 & 7.7061 \\
\hline $\mathrm{Pt}$ & 2.00 & & 3.3363 & & 1.0054 & 1.1408 & 1.0814 \\
\hline $\mathrm{Ta}$ & 2.84 & & 1.6546 & & 7.0805 & 2.5860 & 5.1039 \\
\hline
\end{tabular}

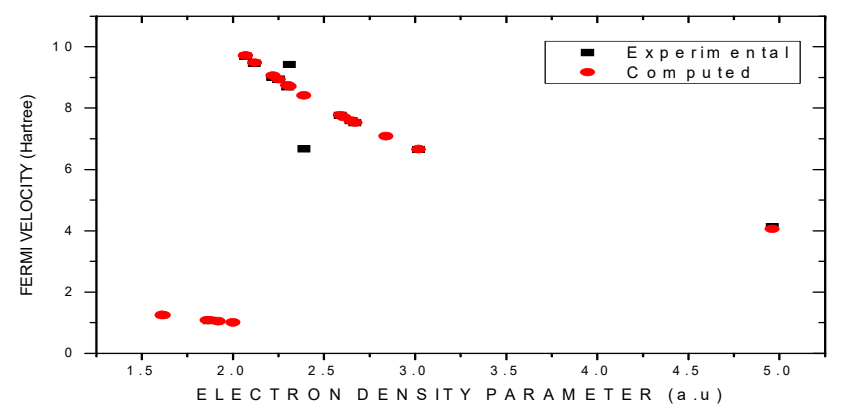

Fig. 1: Variation of Fermi Velocity with Electron Gas Parameter for Some Metals 

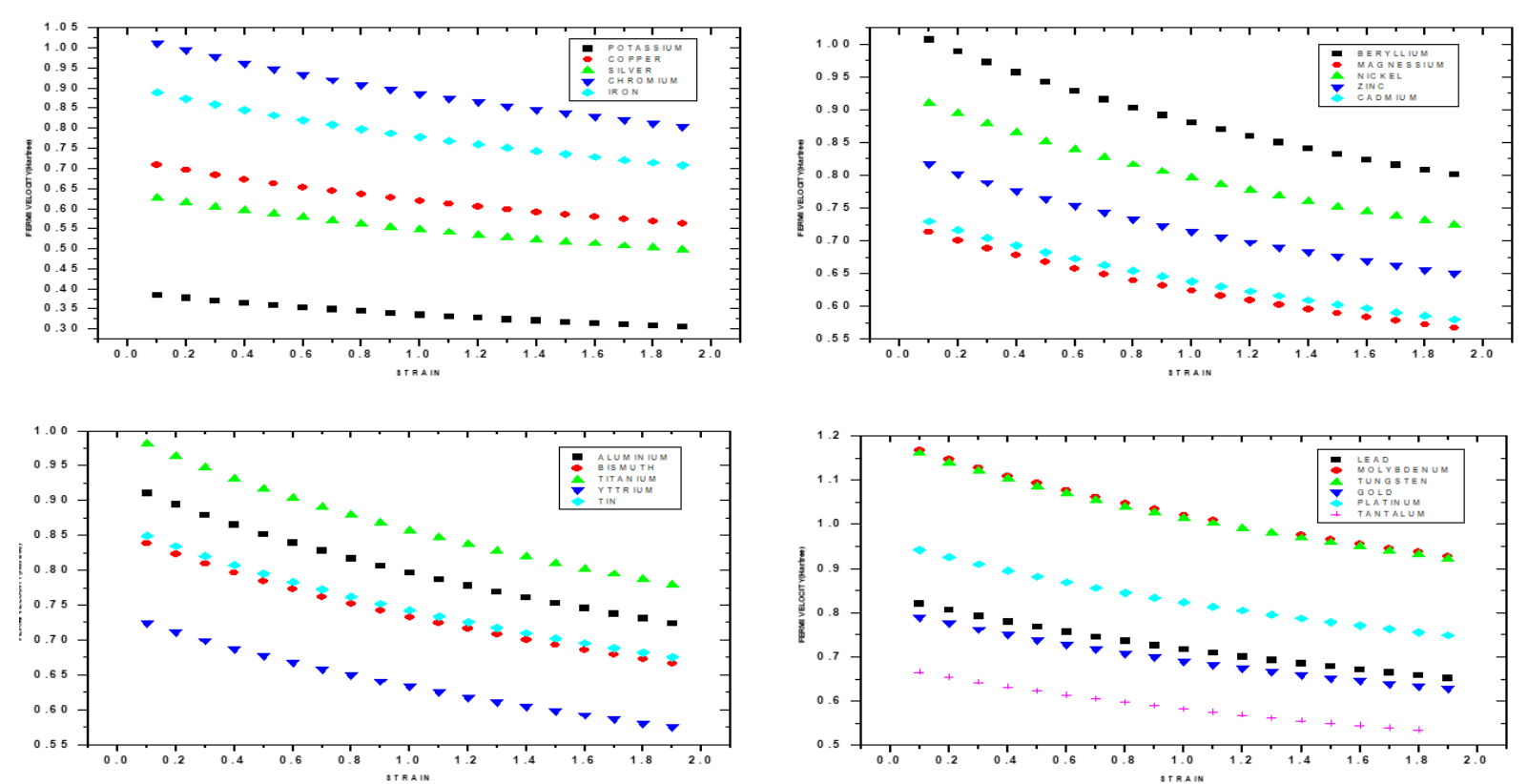

Fig. 2: Variation of Fermi Velocity with Strain for Some Metals

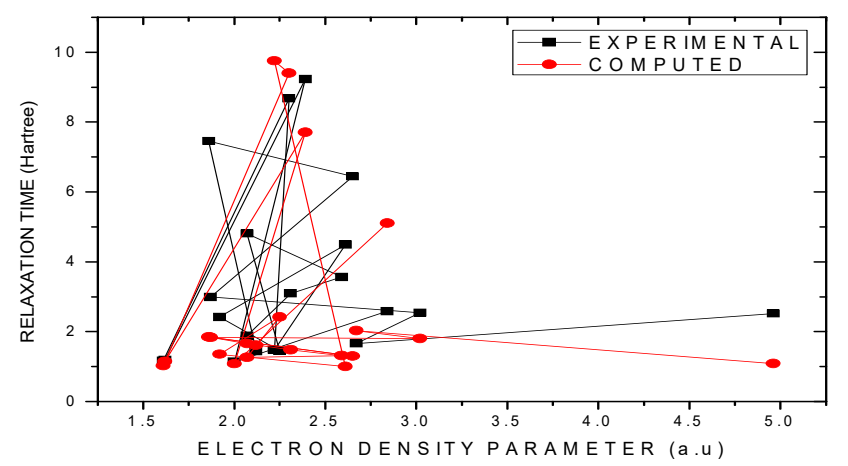

Fig. 3: Variation of Relaxation Time with Electron Gas Parameter for Some Metals
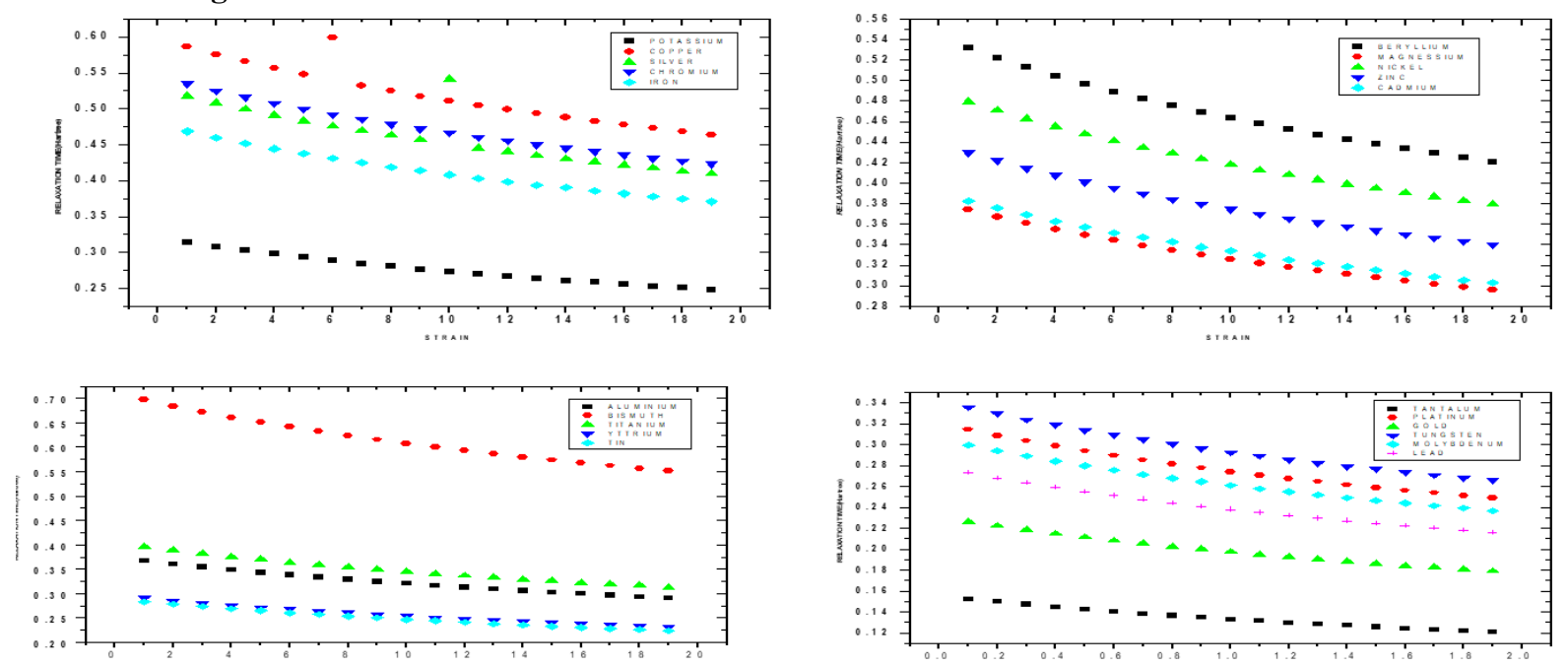

Figure 4: Variation of Relaxation Time with Strain for Some Metals 


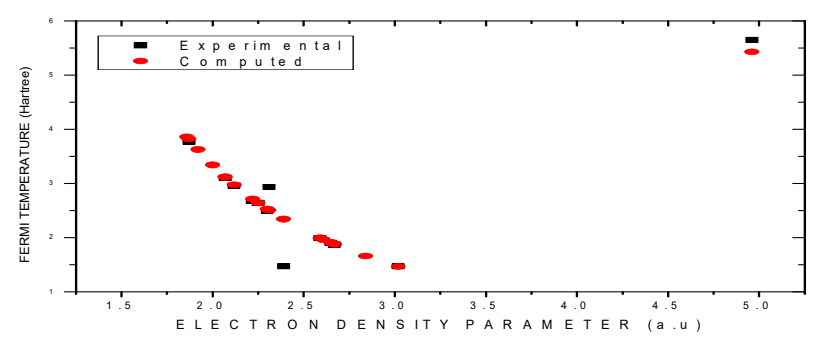

Fig. 5: Variation of Fermi Temperature with Electron Gas Parameter for Some Metals
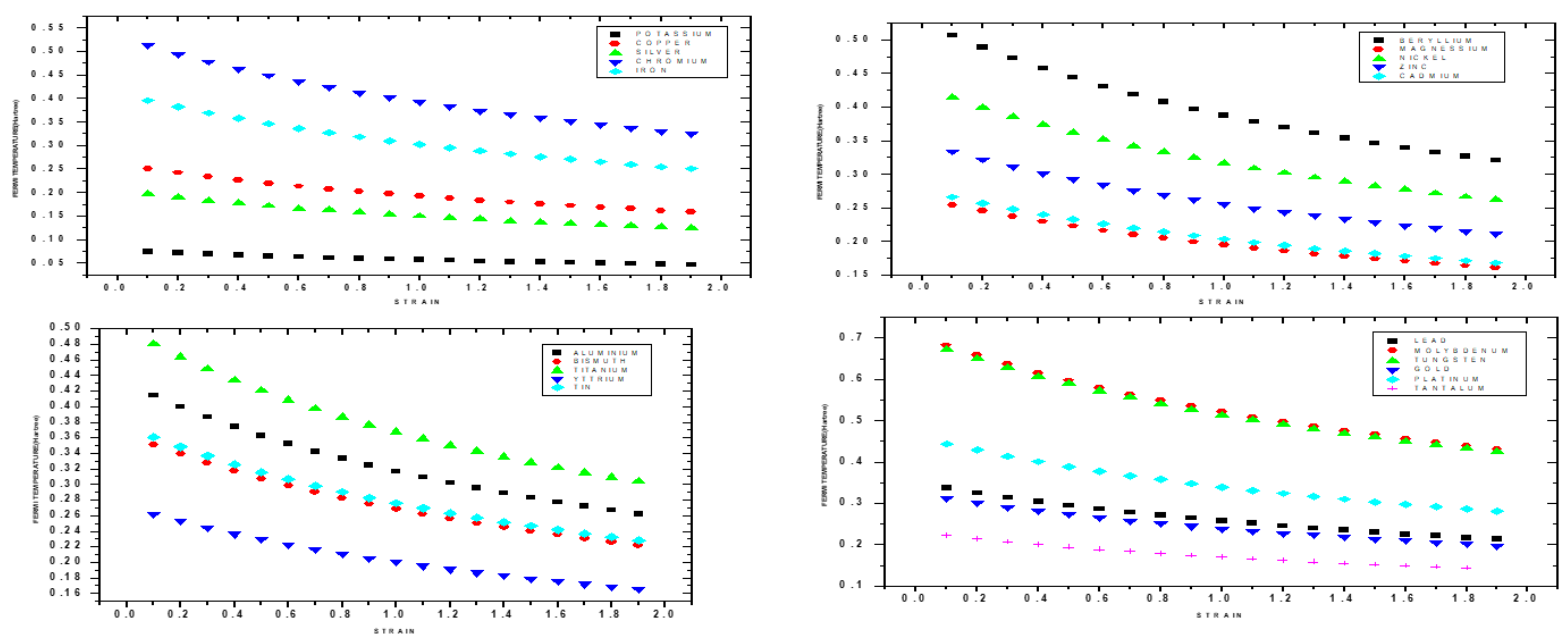

Fig. 6: Variation of Fermi Temperature with Strain for Some Metal

\subsection{Conclusion}

In this work, free electron theory was extended to the study of the effect of linear deformation on Fermi temperature, Fermi velocity and relaxation time of some metals. This study generalized the work of Kiejna and Pogosov (2000) due to the shortcomings of the electron density parameter of deformed metals. This causes the electron density parameter for both deformed and undeformed metals to be equal. A computer programme (FOTRAN 95) was developed and used in the computation of some properties of the selected metals. The result obtained revealed that the relaxation time, Fermi temperature and Fermi velocity of metals decreases with an increase in deformation. This seems to suggest that as deformation increases in a metal the electron interaction decreases and therefore reduces the collision between electrons in the metals. The relaxation time of all the metals investigated decreases as deformation increases. This seems to suggest that as deformation increases the collision probability per unit between the electron decreases as a result of increase in the inter-atomic spacing between the electrons in the metals. The Fermi temperature of metals decreases as deformation increases with the metals in the high density region have high Fermi temperature while metals in the low density region have low Fermi temperature. This suggest that the smaller the electron density concentration the smaller the Fermi temperature. The wavelength of electron at Fermi level increases as deformation increases. This suggest that the higher the electronic concentration in metals the lower the effect of deformation on the wavelength of electron at Fermi level.

\section{References}

1. Adeshakin and Osiele, O.M. (2012). Surface Energy and Surface Stress of Deformed Metals. . Journal of the Nigerian Association of Mathematical Physics, Vol. 21, 421-428.

2. Adesakin, G.E.,Osiele, O.M. and Oluyamo, S.S. (2012). Effects of Deformation on the Energies of Metals. Advances in Physics Theories and Applications. Vol. 10, 11-19.

3. Animalu, A.O.E. (1977).Intermediate quantum Mechanics of crystalline solids. Eagle wood Cliffs, New Jersey.

4. Ashcroft N.W. and Mermin D.N. (1976): Solid State Physics. Holt. Rinchart and Winston. New York.

5. Backofen, W. A. (1972): deformation processing additson - Wesley publishing company. Massachusetts institute of technology.

6. Busch, H. and Schade, H. (1976): Lectures on solid state physics. Zurish, Switzerland.

7. Elliott, S.R. (1997): The Physics and Chemistry of Solids. Trinity college, University of Cambridge UK.

8. Kachhava, C.M. (1992): Solid State Physics. India: Tata McGraw Hill Publishing Company Ltd. 
9. Kiejna A. and Pogosov V.V. (1999): Simple theory of elastically deformed metals: Physical Review, Vol.62, 10445-10450.

10. Kittel C. (1976): Introduction to solid state Physics. Fifth edition. John Wiley and sons. Inc. New York.

11. Madelung, O. (1995): Introduction to Solid State Theory ( $2^{\text {nd }}$ Springer series in Solid State Science). Publish by Spriger. Verlag Berlin Heidelberg.

12. Osiele O. M. and Olubosede (2007) Stabilized Jellium Model Derived Surface Stress of Metals. Journal of the Nigerian Association of Mathematical Physics, Vol. 20, 6-10.

13. Osiele O. M. and Edenma O. G. (2009) Bulk Modulus of Metals According to Structureless Pseudopotantial Model.Journal of the Nigerian Association of Mathematical Physics, Vol. 14, 197-204.

14. Perdew J. P., Tran H. Q. and Smith E. D. (1990): Stabilized jellium: Structureless pseudopotential model for the cohesive and surface properties of metals. Physical review, Vol.42, 11627-11636.

15. Pogosov V.V. and Shtepa O.M. (2006): Effect of deformation on surface characteristics of finite metallic crystals. Zaporozhye, 69064, Ukraine 1065-1071.

16. Pillai S. O. (2010): Solid State Physics. Sixth edition. New Age International Publisher. India, Delhi.

17. Pines D. (1964): Elementary Excitations in solids, Wiley, New York. 